\title{
Evaluation of the system Axima/Saramis MALDI-TOF MS bioMérieux in a clinical laboratory
}

\author{
Romano Mattei', Claudia Vicario², Maria Nardone', Arnaldo Savarino' \\ I Laboratorio Analisi Chimico-Cliniche e Microbiologiche, Ospedale Campo di Marte, Lucca. \\ 2 bioMérieux Italia Spa
}

Key words: Mass Spectrometry, Axima/Saramis, Microbial Identification

\section{Valutazione del sistema MALDI-TOF MS Axima/Saramis bioMérieux in un laboratorio clinico}

\section{SUMMARY}

Introduction. Mass Spectrometry technology "Matrix-assisted laser desorption lonisation/Time-of-Flight (MALDI-TOF MS)" offers a promising new approach for identification of bacteria and fungi of clinical interest. The objective of this study is to evaluate the comparison of microbial identifications obtained with the system Axima/Saramis bioMérieux and identifications obtained by conventional manual or automatic (Vitek 2bioMérieux). The work was divided into a pilot phase in which the staff has been trained and are set instrument parameters and an operational phase.

Methods. MALDI-TOF is a system for the detection and identification of species-specific highly conserved proteins (ribosomal DNA and ligands). Part of a bacterial colony, is deposited on a polymeric substrate, extracted by the addition of the matrix (acid $\alpha$-Cyano-4-hydroxycinnamic) and analyzed by MALDI-TOF.The mass spectra obtained are compared with the database Saramis (Anagnostec) containing the reference spectra of common pathogens.

Results. During the pilot phase were analyzed 303 microorganisms ( $28 \mathrm{I}$ bacteria and 22 fungi), of which 207 $(68.3 \%)$ correctly identified to species level, 35 (1I.6\%) identified as a species but not the main one and 49 (16.2\%) did not identified. In the second phase of the study were analyzed microorganisms I4I7 (134I bacteria and 76 fungi) in a period of 60 days in 1240 (87.5\%) correctly identified to species level, I 2 I (8.5\%) identified as a species but not the main one and $4 \mathrm{I}(2.9 \%)$ unidentified.

Conclusions. By reducing the response time ( $15 \mathrm{~min} / \mathrm{I}$ sample), the use of MALDI-TOF system, bioMérieux facilitates the workflow in the clinical laboratory, and positive impact on length of stay and costs. In addition, the rapid identification of yeasts at the species level is definitely a value added important and independent execution of test sensitivity. Finally, a good operator training translates into improved overall system performance.

\section{INTRODUZIONE}

La Spettrometria di Massa con tecnologia "MatrixAssisted Laser Desorption Ionisation/Time-ofFlight (MALDI-TOF MS)" offre un nuovo e promettente approccio per l'identificazione di batteri e funghi di interesse clinico $(1,2,4)$.

Obiettivo del presente studio è la valutazione del confronto tra identificazioni microbiche ottenute con il sistema Axima/Saramis bioMérieux e identificazioni ottenute con sistemi convenzionali, manuali o automatici (Vitek 2-bioMérieux).

Il lavoro è stato suddiviso in una fase pilota in cui è stato addestrato il personale e settati i parametri dello strumento ed una fase operativa.

\section{METODI}

MALDI-TOF è un sistema per la rilevazione ed identificazione di proteine specie-specifiche altamente conservate (ribosomiali e leganti DNA).
Parte di una colonia batterica, viene depositata su un supporto polimerico, estratta tramite l'aggiunta della matrice (acido $\alpha$-Cyano-4-idrossicinnamico) ed analizzata al MALDI-TOF.

Gli spettri di massa ottenuti sono confrontati con il database Saramis (Anagnostec) contenente gli spettri di riferimento di patogeni comuni.

\section{Preparazione piastrine}

Una piccola quantità di cellule intere di una singola colonia è depositata direttamente in uno spot della piastrina (Figura I).

Viene quindi aggiunto $1 \mu \mathrm{l}$ di matrice CHCA ( $\alpha$-cyano-4-hydroxycinnamic acid).

Per lieviti, aggiunta di acido formico $(25 \%)$ direttamente sulla piastrina.

Ciascuna piastrina ha anche disponibili 3 spot per il calibratore.

\section{Corresponding author: Romano Mattei}

Laboratorio Analisi Chimico e Microbiologiche, Ospedale Campo di Marte

Via dell'Ospedale - 55I00 Lucca - Tel.: 0583 9703 I3; Fax: 0583970402

E-mail:r.mattei@usl2.toscana.it 


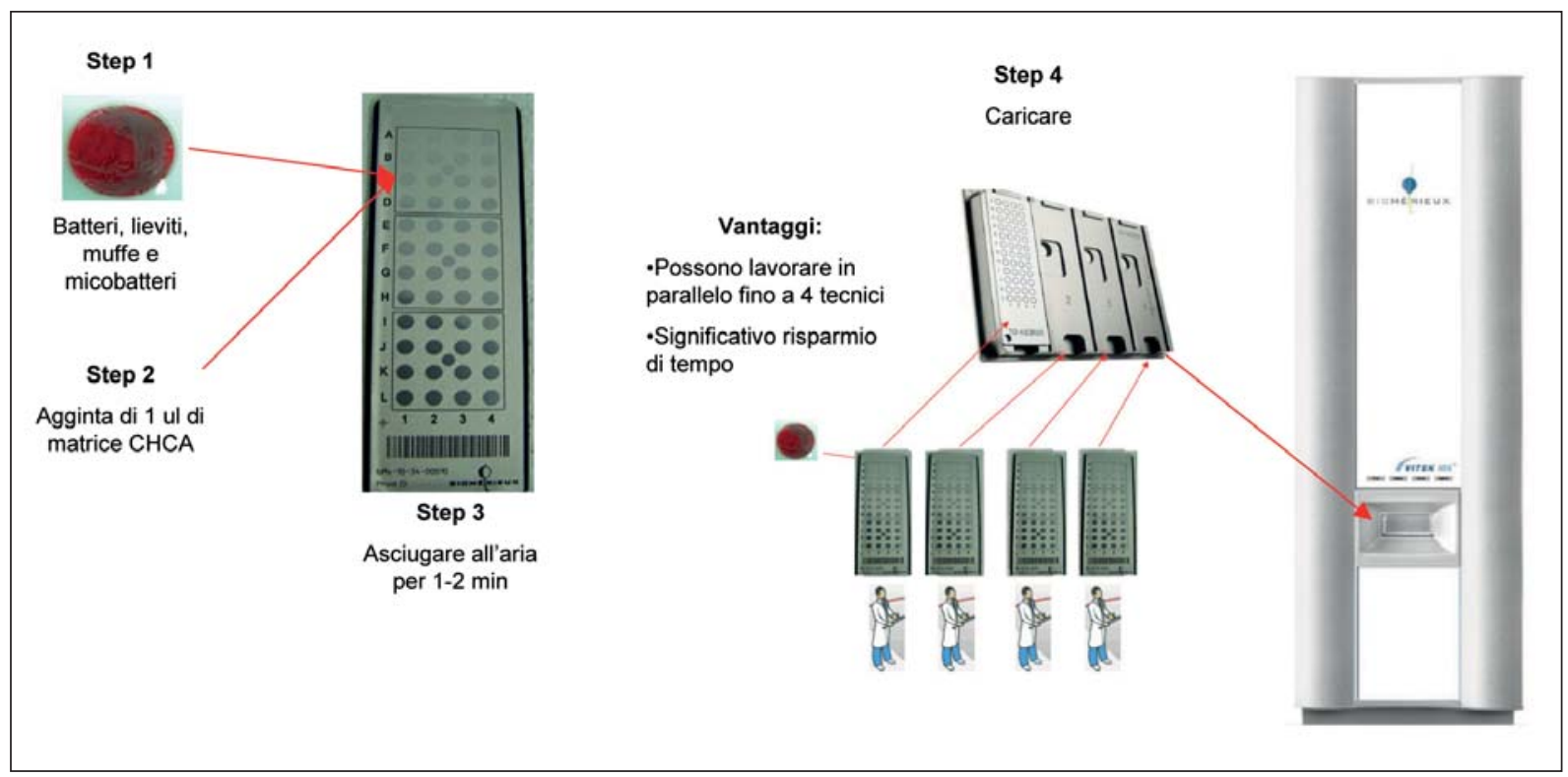

Figura I. Acquisizione degli spettri di massa.

Rilevazione in modalità lineare positiva ad una frequenza del laser di $50 \mathrm{~Hz}$ ed un range di Massa da 2.000-20.000 Da. Voltaggio di Accelerazione e di estrazione $20 \mathrm{kV}$ e tempo di estrazione $200 \mathrm{~ns}$.

$\geq 20$ spari del laser per campione per generare ciascun spettro ionico. Per ciascun campione sono acquisiti e processati 100 spettri di massa di cui è fatta una media per ottenerne lo spettro di massa finale (Figura II).

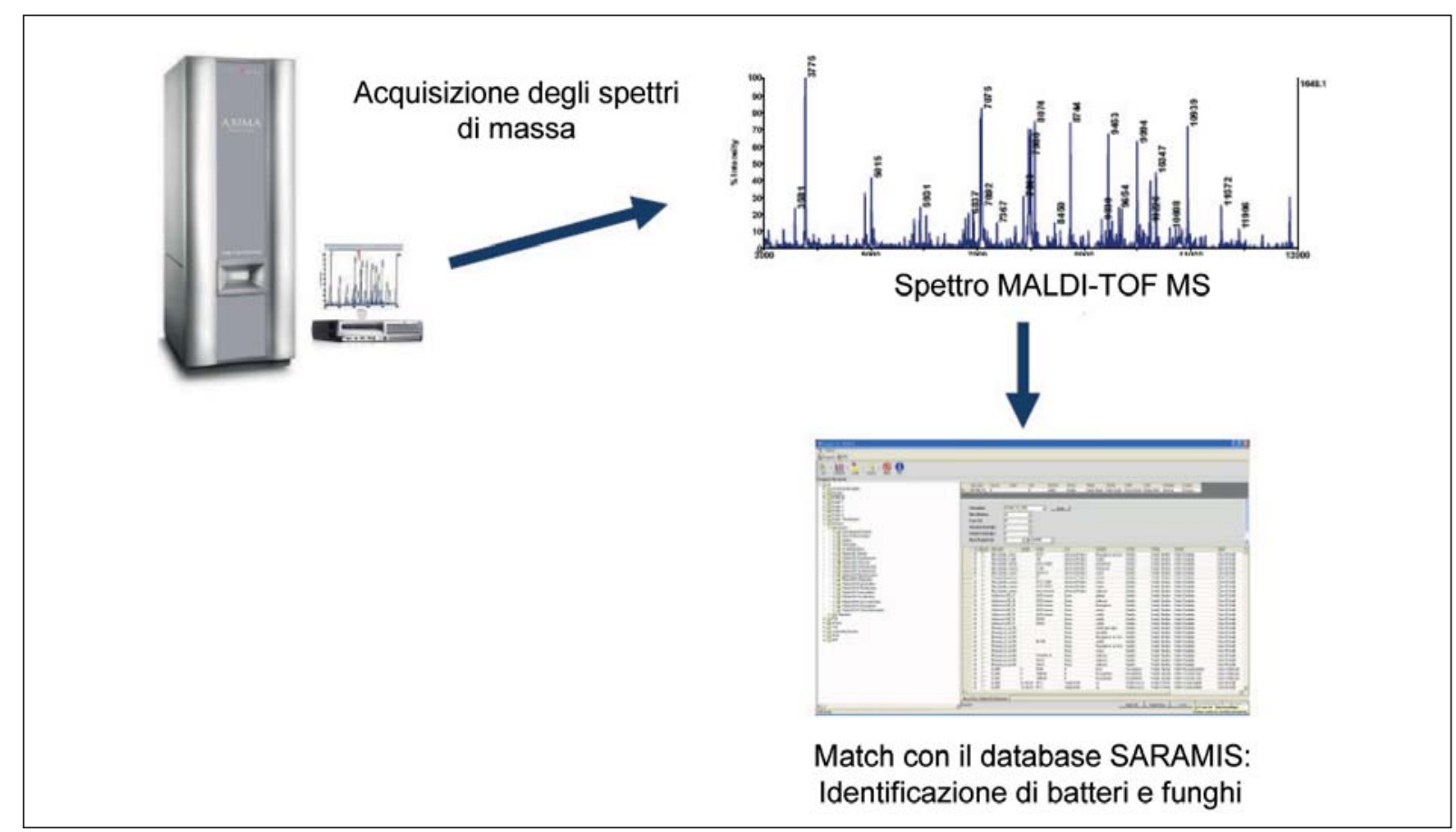

Figura II. Analisi degli spettri di massa ed interpretazione dei risultati

Il database SARAMIS contiene 680 spettri di riferimento genere e specie-specifici (chiamati Superspectra) che includono 540 specie batteriche e 140 fungine. I Superspectra sono stati ottenuti da spettri consensus di spettri di massa multipli di ceppi di riferimento o isolati clinici.

Oltre ai Superspectra il SARAMIS è costituito anche da più di 1400 spettri non eleborati di ceppi di riferimento detti Reference spectra; in questo modo è possibile identificare anche specie atipiche. I risultati dell'identificazione sono visualizzati con un codice colore a seconda della percentuale di identità (dal verde $99.9 \%$ al bianco $70 \%$ ). 


\section{RISULTATI}

Durante la fase pilota sono stati analizzati 303 microrganismi (281 batteri e 22 funghi), di cui 207 (68.3\%) correttamente identificati a livello di specie, 35 (11.6\%) identificati come specie principale ma non unica e $49(16.2 \%)$ non identificati. Nella seconda fase dello studio sono stati analizzati 1417 microrganismi (1341 batteri e 76 funghi) in un periodo di 60 giorni; 1240 (87.5\%) correttamente identificati a livello di specie, 121 $(8.5 \%)$ identificati come specie principale ma non unica e 41 (2.9\%) non identificati (Tabella 1).

\section{CONCLUSIONI}

La drastica riduzione dei tempi di risposta (1.5 minuti/campione), conseguente all'utilizzo del sistema MALDI-TOF AXIMA/SARAMIS bioMèrieux age- vola il flusso di lavoro nel laboratorio clinico e impatta molto favorevolmente sui tempi di refertazione dei risultati.

Questo aspetto si è rivelato particolarmente significativo nel paziente critico in cui a partire dalla coltura primaria è possibile una identificazione di specie in tempo reale.

Nella nostra esperienza è stato così possibile anticipare i tempi diagnostici in numerosi casi di sepsi ed infezioni di liquidi cavitari.

In particolare, si riporta un caso di meningoencefalite da Listeria monocytogenes in cui i test $\mathrm{di}$ biologia molecolare condotti su liquor avevano dato esito negativo e il microrganismo è stato isolato dall'emocoltura ed identificato direttamente dalla subcoltura grazie all'impiego del MALDITOF AXIMA/SARAMIS, con significativo anti-

Tabella I.

FASE PRELIMINARE

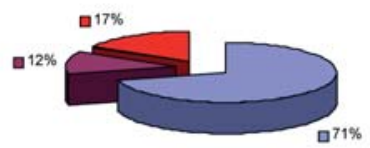

口BUONA ID

aID COME SPECIE PRINCIPALE

aNO ID
FASE OPERATIVA

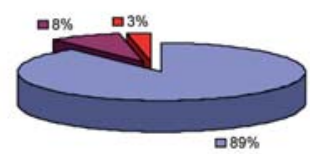

MICETI
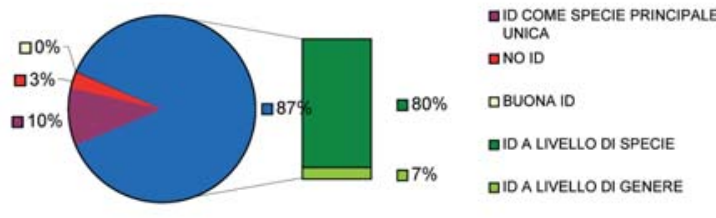

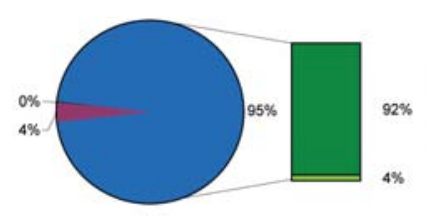

- ID COME SPECIE PRINCIPALE MA NON UNICA
¿BUONA ID

-ID A LIVELLO DI SPECIE

IIDA LIVELLO DI GENERE

\begin{tabular}{|c|c|c|c|c|c|c|c|c|c|}
\hline \multicolumn{3}{|c|}{ Numero di } & \multirow{2}{*}{\multicolumn{2}{|c|}{ Gruppo di organismi }} & \multirow[b]{2}{*}{$\%$ ID TOTALI } & \multicolumn{2}{|c|}{ \%ID a livello di } & \multirow{2}{*}{$\begin{array}{c}\% \text { ID specie } \\
\text { non unica }\end{array}$} & \multirow{2}{*}{$\begin{array}{c}\% \\
\text { NO ID }\end{array}$} \\
\hline famiglia & genere & specie & & & & genere & specie & & \\
\hline & 2 & 13 & 17 & dermatofiti & 88,3 & 11,7 & 76,4 & 11,7 & \\
\hline & 1 & 8 & 9 & funghi filamentosi & 100 & 11,1 & 88,8 & & \\
\hline & & 56 & 57 & lieviti & 98,2 & & 98,2 & 1,7 & \\
\hline & 3 & 77 & 83 & MICETI tot & 96,4 & 3,6 & 93 & 3,6 & \\
\hline & & 10 & 12 & ANAEROBI & 83,3 & & 83,3 & 17 & \\
\hline & 1 & 9 & 11 & cocchi gram negativi & 91 & 9,0 & 82 & 9 & \\
\hline 23 & 35 & 246 & 401 & enterobatteri & 76 & 8,7 & 61 & 19 & 5,2 \\
\hline 2 & 4 & 131 & 151 & gram negativi non fermentanti glucosio & 91 & 2,6 & 86,7 & 5,3 & 4 \\
\hline & & 6 & 6 & campylobacter & 100 & & 100 & & \\
\hline & 7 & 12 & 22 & haemophilus & 86,4 & 32,0 & 54,5 & 13,6 & \\
\hline 25 & 47 & 404 & 591 & BACILLI GRAM NEGATIVI tot & 80,5 & 8,0 & 70 & 15 & 4,5 \\
\hline & 17 & 148 & 183 & streptococchi & 90 & 9,3 & 81 & 9,3 & 0,5 \\
\hline & 23 & 276 & 318 & enterococchi & 94 & 7,2 & 87 & 3 & 3 \\
\hline & 1 & 190 & 205 & stafilococchi & 93 & 0,5 & 93 & 5,4 & 1,5 \\
\hline & & 3 & 6 & micrococchi & 50 & & 50 & 33 & 17 \\
\hline & 41 & 617 & 712 & COCCHI GRAM POSITIVI tot & 95,6 & 5,7 & 87 & 5,4 & 2 \\
\hline & & 2 & 2 & bacillus & 100 & & 100 & & \\
\hline & & 4 & 4 & listeria & 100 & & 100 & & \\
\hline & 1 & 5 & 6 & corinebatteri & 100 & 16,6 & 83,3 & & \\
\hline & & 3 & 3 & lattobacilli & 100 & & 100 & & \\
\hline & 1 & 14 & 15 & BACILLI GRAM POSITIVI tot & 100 & 6,6 & 94 & & \\
\hline 25 & 89 & 1045 & 1330 & BATTERI tot & 87 & 6,7 & 78,5 & 9,7 & 3 \\
\hline 25 & 92 & 1122 & 1413 & TUTTI & 87,6 & 6,5 & 80 & 9,3 & 3 \\
\hline
\end{tabular}


cipo del tempo di risposta.

Inoltre, la possibilità di ottenere una più rapida ed accurata identificazione dei lieviti a livello di specie con questo sistema rappresenta sicuramente un valore aggiunto importante indipendentemente dall'esecuzione del test di sensibilità.

Infine, un buon supporto organizzativo sull' addestramento degli operatori e tecnico sull'avviamento del sistema si traduce nel massimo sfruttamento delle potenzialità del sistema stesso. È auspicabile un'evoluzione del sistema che consenta l'interfacciamento con il Vitek 2 in modo da facilitare il flusso delle fasi operative relative all'identificazione con MALDI-TOF e all'allestimento dell'antibiogramma su VITEK 2. Infine, viste le notevoli potenziali applicazioni del sistema, è altamente auspicabile l'aggiornamento costante del data-base degli spettri e la possibilità di procedere alla identificazione direttamente dai flaconi dell'emocoltura.

\section{BIBLIOGRAFIA}

1. Benagli C, Rossi V, Dolina M, Tonolla M, Petrini O. Matrix-assisted laser desorbition ionizationtime of flight mass spectrometry for the identification of clinically relevant bacteria. PLOS One, 2011; 6 (1).

2. Bizzini A, Durussel C, Bille J, Greub G, Prod'hom G. Performance of matrix-assisted laser desorbition ionization-time of flight mass spectrometry for identification of bacterial strains routinely isolated in a clinical microbiology laboratory. Journal of clinical microbiology, 2010; 1549-54.

3. Cherkaoui A, Hibbs J, Emonet S, et al. Comparison of two matrix-assisted laser desorption ionizationtime of flight mass spectrometry methods with conventional phenotypic identification for routine identification of bacteria to the species level. J Clin Microbiol. 2010; 48 (4): 1169-75.

4. Welker M, Moore ER. Applications of whole-cell matrix-assisted laser desorption/ionization time-offlight mass spectrometry in systematic microbiology. Syst Appl Microbiol. 2011; 34 (1): 2-11. 